\title{
The Diagnostic Role of p53 and Ki 67 Immunohistochemistry in Evaluation of Urinary Bladder Carcinomas in Egyptian Patients
}

\author{
Sahar Ali Mohamed \\ Department of Pathology, General Organization of Teaching Hospitals and Institutes (GOTHI), Cairo, Egypt \\ Email address: \\ Saharali.65@hotmail.com \\ To cite this article: \\ Sahar Ali Mohamed. The Diagnostic Role of p53 and Ki 67 Immunohistochemistry in Evaluation of Urinary Bladder Carcinomas in \\ Egyptian Patients. International Journal of Chinese Medicine. Vol. 3, No. 1, 2019, pp. 1-6. doi: 10.11648/j.ijcm.20190301.11
}

Received: April 23, 2019; Accepted: May 30, 2019; Published: June 10, 2019

\begin{abstract}
Background: Urothelial carcinomas (UC) are the most common cancers of urinary bladder responsible for a significant proportion of cancer morbidity and mortality. In Egypt, bladder cancer is the second most common malignancy. We did the present investigation to exhibit the clinicohistopathological highlights and to associate the p53 and Ki 67 immunoexpression with grade and stage of bladder carcinomas. Materials and Methods: sixty-two cases of bladder neoplasm were investigated. Grading and staging was done. An immunohistochemical assay for $\mathrm{p} 53$ and $\mathrm{Ki} 67$ was performed in altogether cases, with relation to clinicopathological parameter. Results: There was a significant association of p53 and Ki 67 overexpression in high grade with the epithelial type $(\mathrm{P}=0.0001) \&(\mathrm{P}=.0 .01)$ respectively. While there was a statistically significant association of high expression of P53 in PT1 compared to PTa and PT2 $(\mathrm{p}=0.000001)$. There was a significant correlation between Ki 67 in staging $\mathrm{pT} 1(\mathrm{pTa}+\mathrm{pT} 1 / \mathrm{pT} 2, \mathrm{P}=0.000001 ; \mathrm{pTa} / \mathrm{pT} 1, \mathrm{P}=0.000001 ; \mathrm{pT} 1 / \mathrm{pT} 2, \mathrm{P}=0.000002)$. Conclusion: The role of $\mathrm{p} 53$ and Ki 67 immunohistochemical relativities in the differential diagnosis and prognosis of urinary bladder carcinomas in step with the pathological stage and histologic grade of the neoplasm, will be understood due to unique biology and pathogenic features of these tumors. Thus, this study supports the opinion that combined use of p53 and Ki 67 immunostaining markers may help as further prognostic clue alongside with histologic grading and staging in bladder carcinomas.
\end{abstract}

Keywords: Urinary Carcinomas, p53, Ki 67, Urothelial, Prognosis, Stage, Grade

\section{Introduction}

Bladder cancer (BC) may be a heterogeneous disease. It is the most frequent urinary tract tumor [1-2]. In 2017, more or less seventy-nine thousand patients worldwide is going to be diagnosed with the malignant neoplastic disease of the urinary bladder [2]. Cystoscopy, urinary cytology and transurethral resection (TUR) are utilized to predict the diagnosis and follow-up in $\mathrm{BC}$ [3-4]. As prognostic factors for aggressiveness in $\mathrm{BC}$ [3], histological grade and TNM staging are necessary. However, there is no definite parameter to predict $\mathrm{BC}$ 's behavior and prognosis due to $\mathrm{BC}$ heterogeneity [5-6].

P53 and Ki 67 expressions have frequently used urothelial malignancy enzyme labels. P53 is a key tumor suppressor gene involved in genomic balance, genotoxic stress response, and cell cycle apoptosis stimulation. Some studies showed correlations in urothelial bladder cancer between p53 and tumor stage and grade [7-9].

In Egypt, bladder cancer is the second most common malignancy and constitute a costly problem for the Egyptian patients [10]. Indeed, this study updated the clinicopathological characteristics of bladder carcinomas and showed the correlation of p53 and Ki 67 with grade and stage of bladder carcinomas at the initial presentation in Egyptian population.

\section{Materials and Methods}

\subsection{Patients and Specimens}

We included a total of 62 cases of urinary bladder carcinomas diagnosed over the past five years in the present study. The study has been reviewed and approved by the Institutional Ethical Committee.

Tissue samples were received in $10 \%$ buffered formalin, as 
TURBT chips or bladder biopsy in histopathology sections and processed. 3-5 $\mu$ thick, $\mathrm{H}$ and $\mathrm{E}$ stained sections for histopathological assessment.

Patients were randomly selected on the basis of tissue availability. Clinical data including patient age, microscopic hematuria, lower abdominal pain, tumor grade, and stage were extracted from the hospital computerized database and patient records. Then they were revised, reassessed and graded as reported by the guidance of the WHO-2004, and were staged according to the TNM classification of the American Joint committee of cancer.

\subsection{Tissue Microarray Construction}

A manual tissue microarray (TMA) was performed using a mechanical pencil tip method of Shebl et al. [11] and Soliman and Yussif [12]. Cores from a tissue of breast carcinoma and chronic tonsillitis were also taken as a positive internal control for p53 and Ki 67 respectively.

\subsection{Immunohistochemistry}

Extra 2-4 $\mu$ thick sections were taken on Poly-L lysine coated slides. IHC examination was performed using a Ventana Benchmark Ultra machine automated staining system. The primary antibody used was p53 and Ki 67 (clone DO-7, rabbit monoclonal primary antibody) (Ventana, Tucson, AZ, USA). A check was performed for accuracy through positive internal controls for $\mathrm{p} 53$ in sections of breast carcinoma tissue and chronic tonsillitis tissue for Ki 67. For negative controls, the primary antibody was substituted with phosphate buffer saline in duple sections.

Nuclear positivity was seen as dark brown color on the faint bluish background. Percentage of immunopositive cells was determined by counting at least 1000 tumor cells in areas of maximum positivity. The results were determined as taking the cutoff value as $20 \%$ and split into three categories as immunonegative, $<20 \%$ as low, and $>20 \%$ as a high expression for both immunomarkers.

\subsection{Statistical Analysis}

The data were subsequently studied, tabulated and correlated with the diagnosis of histopathology. Statistical analysis was performed using Chi-square test and Fisher's exact test by using the statistical package for social science SPSS version 20 . P values of 0.05 or less were considered statistically significant.

\section{Results}

\subsection{Clinicopathologic Features}

Most of the patients were males 49 cases (79\%) and this study reported only 13 cases $(21 \%)$ in females with a male to female ratio of 3.7:1. The median age of male presentation was 54 years, while it was 61 years in females.

The age group of 71-80 years ( 22 cases, $35.5 \%$ ) had the maximum number of cases followed by the age group of 51-
60 years (17 cases, $27.4 \%$ ). No case up to the age of 20 has been reported. The age group of 71-80 years was mostly male patients (17 cases), while female patients in that group were only 5 cases (Table 1).

Clinically, the most common presentation in my study was in 57 cases $(91.9 \%)$ of gross or microscopic hematuria. Hematuria alone was observed in 22 cases $(35.5 \%)$, while its association with symptoms of the urinary tract or lower abdominal pain was observed in 35 cases $(56.4 \%)$ and 8 cases $(12.9 \%)$, respectively. There were just 5 cases of UTI and 2 cases of lower abdominal pain alone. Only one patient was asymptomatic and diagnosed by chance.

\subsection{Histopathologic Features}

The most common histological variant was urothelial carcinoma seen in 60 cases $(96.8 \%)$, followed by squamous cell carcinoma ( 1 case) and adenocarcinoma (1 case). All cases were classified as low (LG) and high grade (HG) according to WHO-2004 recommendations. Thirteen cases (21\%) were reported as LG, while the remaining 49 cases (79\%) were reported as HG tumors, including squamous carcinoma (Figure 2a) and adenocarcinoma. All cases were also staged in accordance with the American Joint CancerTNM Classification Committee.

In this study, 45 patients $(72.6 \%)$ revealed nonmuscle invasive bladder carcinoma, of which 13 cases $(21 \%)$ had a stage pTa (without lamina propria invasion) and 32 cases $(53.2 \%)$ were of stage pT1 (with lamina invasion) whereas the remaining 17 cases $(25.8 \%)$ showed stage with pT2 (detrusor muscle-invasive disease) (Table 1). Patients with stage T1 were further classified as pT1 HG (14 cases) and pT1 LG (18 cases) according to their grade.

Table 1. Distribution pattern of bladder carcinoma patients, according to age, gender, histologic type, grade, and stage of bladder tumors.

\begin{tabular}{lll}
\hline Characteristic & Male & Female \\
\hline Total cases & 49 & 13 \\
Age Group (years) & & \\
$40-50$ & 1 & 3 \\
$51-60$ & 12 & 5 \\
$61-70$ & 17 & 0 \\
$71-80$ & 17 & 5 \\
$>80$ & 2 & 0 \\
Tumor Histology & & \\
Urothelial cell carcinoma & 47 & 13 \\
Squamous cell carcinoma & 1 & 0 \\
Adenocarcinoma & 1 & 0 \\
Pathologic Grade & & \\
Low & 7 & 6 \\
High & 42 & 7 \\
Stage & & \\
PT a & 9 & 4 \\
PT 1 & 28 & 4 \\
PT 2 & 12 & 5 \\
\hline
\end{tabular}

\subsection{Immunohistochemical Features}

\subsubsection{Ki 67 Expression}

Low immunoexpression of $\mathrm{Ki} 67$ in 29 cases and high expression in 33 patients were noted in this study. LG tumors 
showed predominantly a low expression (11 cases) and HG tumor showed a high expression (2 cases) (Figure 1b) and (Figure 1d). All 17 cases of muscle-invasive tumors (pT2) showed high levels of immune expression of $\mathrm{Ki} 67$ including squamous cells and adenocarcinoma (Figure 2c) and (Figure 2f).

While all patients with pTa stage showed low expression.
In pT1 staged carcinomas, low and high expression was noticed in 19 and 13 cases respectively. A difference was found to be statistically significant in terms of grading $(\mathrm{P}=$ $0.01 ; \mathrm{df}=1)$ and staging $\mathrm{pT} 1(\mathrm{pTa}+\mathrm{pT} 1 / \mathrm{pT} 2, \mathrm{P}=0.000001$; $\mathrm{pTa} / \mathrm{pT} 1, \mathrm{P}=0.000001 ; \mathrm{pT} 1 / \mathrm{pT} 2, \mathrm{P}=0.000002 ; \mathrm{df}=1)$ highlighted in (Table 2).

Table 2. Distribution pattern of ki 67 immunohistochemical staining, according to histology, grade, and stage.

\begin{tabular}{|c|c|c|c|c|}
\hline Ki 67 & Immunonegative & $<20 \%$ & $>20 \%$ & $\mathbf{p}$ \\
\hline Total cases & 0 & 29 & 33 & \\
\hline \multicolumn{5}{|l|}{ Tumor Histology } \\
\hline Urothelial carcinoma & 0 & 29 & 31 & \\
\hline Squamous cell carcinoma & 0 & 0 & 1 & \\
\hline Adenocarcinoma & 0 & 0 & 1 & \\
\hline \multicolumn{5}{|l|}{ Pathologic Grading } \\
\hline Low & 0 & 11 & 2 & 0.01 \\
\hline High & 0 & 18 & 31 & \\
\hline \multicolumn{5}{|l|}{ Stage } \\
\hline PTa & 0 & 11 & 2 & \\
\hline PT1 & 0 & 18 & 14 & \\
\hline PT2 & 0 & 0 & 17 & 0.000001 \\
\hline
\end{tabular}

\subsubsection{P53 Expression}

It was seen that, out of 62 cases of urinary bladder carcinoma, 42 cases $(67.7 \%)$ showed high p53 expression, 17 cases $(27.5 \%)$ showed low p53 expression, and 3 cases $(4.8 \%)$ were immunonegative for $\mathrm{p} 53$. High expression of p53 was seen mainly in HG tumors (26 cases) as compared to 16 cases of LG tumors (Figure 1a) and (Figure 1c).

Low expression was noticed in 9 patients of LG tumors and in only 8 cases of HG tumors. This difference of p53 expression was statistically significant $(\mathrm{P}=0.0001 ; \mathrm{df}=1)$ while taking grading into account. A maximum number of patients with high p53 expression (21 cases) was observed in stage pT1 as compared to stage pTa (8 cases) and pT2 (14 cases).

Only three cases each of pTa and pT2 stage showed low immunoexpression whereas stage pT1 showed 10 cases. In terms of staging, these results were also statistically significant $(\mathrm{P}=0.000001 ; \mathrm{df}=1)$. A case of squamous cell carcinoma showed low p53 expression, whereas, the single case of adenocarcinoma revealed no immunoexpression (Figure 2e) and (Figure 2b). Out of three immunonegative patients, two were of pTa stage and one each of pT1 and pT2 stage including a single case of HG urothelial carcinoma (Table 3).

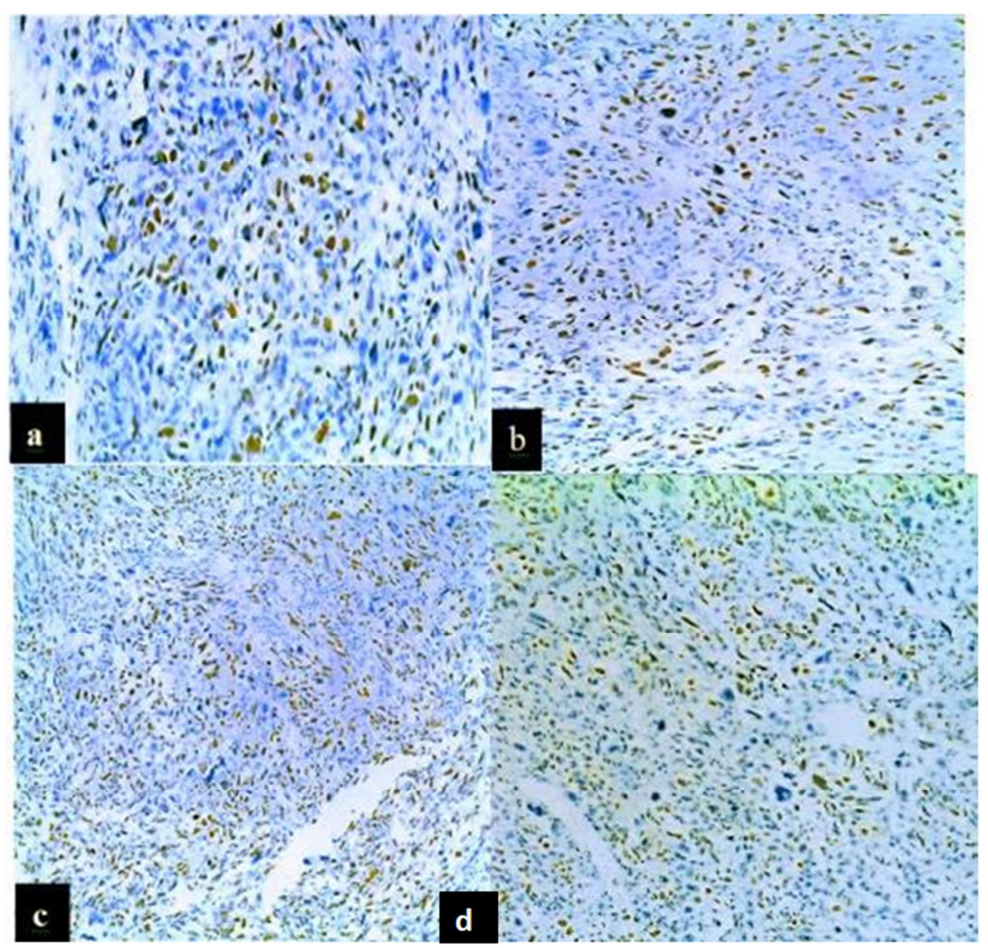

Figure 1. (a\&b) Sections of urothelial carcinoma showing low expression of both $p 53 \&$ Ki67 (x400) respectively. (c\&d) Sections of high expression of p53 \& Ki67 (x100) each respectively. 


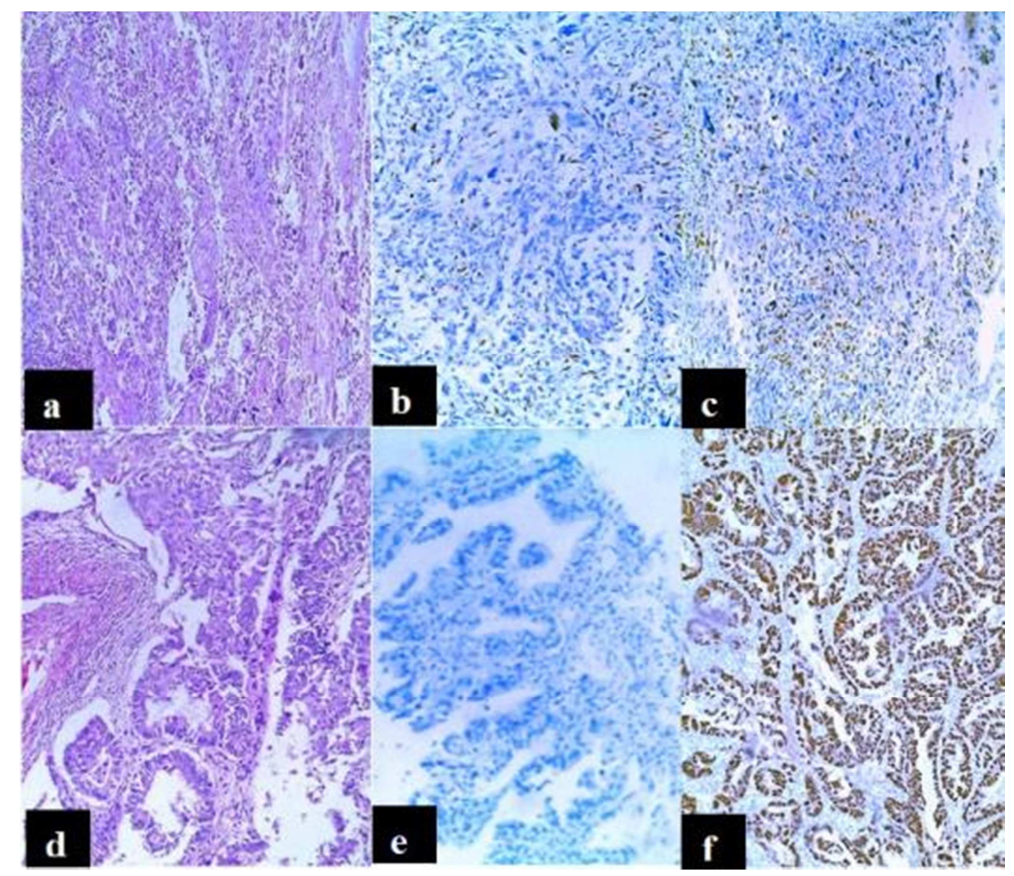

Figure 2. (a) Squamous cell carcinoma (SCC)(H\&E x 100). (b) Low expression of 553 in SCC (IHC, X 100). (c) High expression of Ki 67 in SCC (IHC, X, 100) (d) Adenocarcinoma (AC of urinary bladder (H\&E, X100). (e) Negative expression of p53 in AC (IHC, X 100). (f) High expression of Ki-67 of AC (IH C, X100).

Table 3. Distribution pattern of p53 immunohistochemical staining according to histology, grade, and. Stage.

\begin{tabular}{lllll}
\hline P53 & Immunonegative & $<\mathbf{2 0} \%$ & $\mathbf{2 0 \%}$ & p \\
\hline Total cases & 3 & 17 & 42 & \\
Tumor Histology & 2 & 16 & 42 & \\
Urothelial carcinoma & 0 & 1 & 0 & \\
Squamous cell carcinoma & 1 & 0 & 0 & 0.0001 \\
Adenocarcinoma & 2 & 9 & 16 & \\
Pathologic Grading & 1 & 8 & 26 & \\
Low & 2 & 3 & 8 & \\
High & 1 & 10 & 21 & \\
Stage & 0 & 3 & 14 & \\
PTa & & & \\
PT1 & & & \\
PT2 & & & \\
\hline
\end{tabular}

\section{Discussion}

$\mathrm{BC}$ is a heterogeneous neoplasm that ranks ninth in the worldwide incidence of cancer with a high probability of recurrence and progression, showing completely different rates of metastasis and mortality, betting on tumor grade and staging. Although the incidence of urothelial carcinomas increases with age, smoking, exposure to petrochemicals, and arylamines, its burden also increases in recent decades due to the shift in smoking habits, tobacco use, industrialization, and urbanization from developed countries to developing countries $[1,10,13]$.

However, BC occurs mostly in men, affecting three times the number of men as women, as reported by many authors and guidelines $[1,3,10]$. Our study had a ratio of 3.7 men for each woman belonging to the middle to older age groups. Similar findings was also described in other studies [9, 12-13].

In addition, hematuria was a common symptom that is consistent with different authors' findings [13-15]. $10 \%$ or $20 \%$ of patients with microscopic or gross hematuria are subsequently diagnosed after evaluation as bladder carcinoma [15].

As well as, the predominant histological variant in the present study was urothelial carcinoma. Several authors have also jointly printed similar data $[5,10,13,16-17,20]$. Adenocarcinomas and squamous cell carcinomas are rare tumors of the bladder representing approximately $1.6 \%$ of all carcinomas of the bladder that almost match our study. Beltran et al. [21] jointly represented all vesical tumors as $1 \%-3 \%$ of their incidence.

A reliable and identical staging and grading system based on histopathological analysis for bladder carcinomas is important for categorizing patients for better treatment options between institutions, and also for prognostically stratifying them into separate groups. At first presentation, approximately $80 \%$ of patients are diagnosed as superficial non-muscle invasive carcinoma. In this study, non-muscle 
invasive tumors have been noted in approximately $74 \%$ of the cases (stage $\mathrm{Ta}+\mathrm{T} 1$ ). Similar findings have also been reported in other studies [13, 16-18].

Therefore, the presence of detrusor muscle in the biopsy specimen is also important to mention. Depending on the cell atypia, polarity, epithelial thickness, and mitosis (WHO-2004 grading system), we divided urothelial carcinomas as LG and HG.

As there is an increased risk of tumor recurrence, stage progression, and tumor-related mortality with increasing tumor grade and infiltration patterns, histopathological grading and staging of urothelial carcinomas may be an important tool in determining the disease prognosis [1-2]. However, these pathological variables have limited ability to predict the response to treatment more than where different treatment approaches and follow-up schedules are present. Therefore, there is a clear need for molecular studies that can help predict the behavior of these cases accurately.

Thus, the p53 gene is the most common gene mutation in many tumors such as lung, breast, colon and bladder carcinoma. It is located on chromosome $17 \mathrm{p}$ tumor suppressor, and plays a role in the regulation of cell proliferation and mutation of the cell cycle, so it is responsible for genome stability, response to genotoxic stimuli, and activation of apoptosis [2, 4].

Moreover, anomalies of proliferation resulting from disruption of cell cycle regulators and also abnormal cell proliferation can be easily detected by immunohistochemistry using the $\mathrm{Ki} 67$ labeling index, which is a measure of cell growth fraction and also of biological malignancy aggressiveness. Nuclear antigen Ki 67 coded on chromosome 10 by the gene is absent in resting cells ( $\mathrm{G} 0$ phase) and therefore exclusively positive in proliferating cell nuclei $[1,20]$.

In regards to this study, as this discriminating cutoff value was more predictive in terms of tumor recurrence, progression, and disease-free survival in some of the previous analytical studies, we took $20 \%$ nuclear positivity as the cutoff value for low and high p53 and $\mathrm{Ki} 67$ immunoexpression [13, 24].

The significant combination of grade and stage (superficial versus invasive muscle) with positively stained tumor cells p53 was observed while taking the cutoff value of $20 \%(\mathrm{P}=$ 0.0001 and $\mathrm{P}=0.000001$ ) (Table 3). Some other authors noted similar behavior in the tumor grade and/or stage or clinical outcome of this marker [13, 18, 20].

Qamar et al. found that higher p53 expression can be an indicator of tumor progression and early failure of local therapy, as well as early surgical intervention, especially for superficial carcinomas. Although, the opposite results were also described by some investigators [8-9, 13].

We also observed p53 immunonegative cases, which may indicate the presence of inactivation of p53 or other alternative genetic pathways in the molecular pathogenesis of urothelial tumor. As also suggested by some previous analyzes in alternative immunomarkers $[13,18]$. There was, also, a positive significant correlation of the $\mathrm{Ki} 67$ labeling index with histological grading and staging of urothelial carcinomas ( $\mathrm{P}<0.05)$, similarly described by other studies $[8$, 13, 21]. All muscle-invasive carcinomas revealed high Ki 67 immunoexpression, and no immuno-negative case was observed either.

In our study, the percentage of positively stained p53 tumor cells ranged from 0 percent to $95.1 \%$, and the mean overall p53 expression value was $31.7 \%(\mathrm{pTa}=17.7 \%, \mathrm{pT} 1=$ $50 \%$, pT2 $=27.4 \%$, whereas the median value was $37.5 \%$. The mean Ki 67 labeling index was 33.3\% (pTa $=20.9 \%$; pT $1=51.6 \%$; pT2 $=27.4 \%$ ) and the mean value was $24.2 \%$. We found that Ki 67 's mean expression was changed with staging more and more compared to the expression of $\mathrm{p} 53$. In addition, there was more difference between pTa and pT1 (P $=0.0001)$ expression of these two markers compared to pT2.

\section{Conclusion}

P53 and Ki 67 as cell cycle and proliferation regulative proteins are probably capable of promoting the prognostic ability of directly used histological grading and staging, but because of increasing BC's biological and clinical heterogeneity, one marker is unlikely to predict precise prognostic classes. Consequently, the opinion that combined use of p53 and Ki 67 immunomarkers in carcinomas of the urinary bladder may add further prognostic information along with histological grading and staging. Thus, our work corroborate with few studies $[13,24]$ that had similar results. Unfortunately, due to their referral to higher centers, there was a big problem to follow up with the patients.

\section{Conflict of Interest}

The authors declare that they have no competing interests.

\section{Acknowledgements}

The author gratefully acknowledges all staff members of pathology department of the National Institute of Urology and Nephrology and that of Al Galaa Teaching Hospital.

\section{References}

[1] Mingshan Li, 1 YixiangWang, 1 Yili Liu, 1 Xiling Zhang, 1 Jie Liu, 2 and Ping Wang. Low Expression of hsa_circ_0018069 in Human Bladder Cancer and Its Clinical Significance. Hindawi BioMed Research International, 2019; Article ID 9681863, 9 pages https://doi.org/10.1155/2019/9681863

[2] da Silva, J. N. L., Ranzi, A. D., Carvalho, C. T. et al. Pathol. Oncol. Res. (2018). https://doi.org/10.+1007/s12253-018-0389-5

[3] Siegel RL, Miller KD, and Jemal A. (2017) Cancer Statistics, 2017. CA Cancer J Clin 2017; 67: 7-30es.

[4] Compérat E et al, Comparaison des classifications TNM des 8 éditions de l'UICC et de JCCenuropathologie Comparison of UICC and AJCC 8th edition TNM classifications in uropathology. Annales de Pathologie. 2019; 39 (2): 158-166. 
[5] Kandori S, Kojima T, Nishiyama $\mathrm{H}$. The updated points of TNM classification of urological cancers in the 8th edition of AJCC and UICC, Japanese Journal of Clinical Oncology. 2019; 49 (5): 421-425 https://doi.org/10.1093/jjco/hyz017.

[6] Xylinas E, Kluth LA, Lotan Y, Daneshmand S, Rieken M, Karakiewicz PI et al Blood- and tissue-based biomarkers for prediction of outcomes in urothelial carcinoma of the bladder. Urol Oncol. 2014 32: 230-242.

[7] ElkadyN, Sultan M, Elkhouly E. Evaluation of topoisomerase II, ki-67, and P53 expression in non-muscle-invasive urothelial carcinoma and their clinical significanc. 2018; 61 (4): 526-531.

[8] $\mathrm{Wu} \mathrm{P}$ et al. Low-level Ki-67 expression as an independent predictor of bladder tumour recurrence in patients with primary upper tract urothelial carcinoma after radical nephroureterectomy. Japanese Journal of Clinical Oncology, 2015, 45 (12) 1175-1181 doi: 10.1093/jjco/hyv139.

[9] Qamar S, Inam QA, Ashraf S, Khan MS, Khokhar MA, Awan N. Prognostic Value of p53 Expression Intensity in Urothelial Cancers. J Coll Physicians Surg Pak. 2017 Apr; 27 (4): 232236. doi: 2596.

[10] Kyritsi F, Christopher A. Loffredo, Yun-Ling Zheng, George Philips, and Sania Amr, "Urinary Bladder Cancer in Egypt: Are There Gender Differences in Its Histopathological Presentation?," Advances in Urology, vol. 2018, Article ID 3453808, 7 pages, 2018 https://doi.org/10.1155/2018/3453808

[11] Shebl AM, Zalata KR, Amin MM, El-Hawary AK (2011). An inexpensive method of small paraffin tissue microarrays using mechanical pencil tips. Diagn Pathol, 2011; 6, 117.

[12] Soliman NA and Yussif SM. Ki-67 as a prognostic marker according to breast cancer molecular subtype. Cancer Biology \& Medicine. 2016; 13 (4), 496-504.

[13] Thakur B., Kishore S, Dutta K., Kaushik S. and Bhardwaj A. Role of p53 and Ki-67 immunomarkers in carcinoma of urinary bladder. Indian J Pathol M. 2017; 60: (4); 505-509.

[14] Poyet C, Jentsch B, Hermanns T, Schweckendiek D, Seifert $\mathrm{H}-\mathrm{H}$, Schmidt peter M, Sulser T Et al. Expression of histone deacetylases 1,2 and 3 in urothelial bladder cancer. BMC Clin Pathol 2014; 14: 10.

[15] Paner GP, Stadler WM, Hansel DE, Montironi R, Lin DW, Amin MBUpdates in the Eighth Edition of the Tumor-NodeMetastasis Staging Classification for Urologic Cancers. Eur Urol. 2018; 73 (4): 560-569. doi: 10.1016/j.eururo.

[16] Gupta P, Jain M, Kapoor R, Muruganandham K, Srivastava A, Mandhani A. Impact of age and gender on the clinicopathological characteristics of bladder cancer. Indian $\mathrm{J}$ Urol 2009; 25: 207-10.

[17] Sathya M, Chinnaswamy P. Urinary bladder cancer: A clinicopathological and histological study J Med Sci 2014; 14: 206-9.

[18] Koyuncuer A. Immunohistochemical expression of p63, p53 in urinary bladder carcinoma Indian J Pathol Microbiol. 2013; 56: (1); 10-15.

[19] Geelvink M et al. Diagnostic and Prognostic Implications of FGFR3high/Ki67high Papillary Bladder Cancers. Int. J. Mol. Sci. 2018, 19, 2548; doi: 10.3390/ijms19092548.

[20] Pudasaini S, Subedi N, Prasad KB, Rauniyar SK, Joshi BR, Bhomi KK. Cystoscopic bladder biopsies: A histopathological study. Nepal Med Coll J 2014; 16: 9-12.

[21] Beltran H, Robinson BD, Tagawa ST. Primary squamous cell carcinoma of the urinary bladder presenting as peritoneal carcinomatosis. Adv Urol 2010; Vol. 2010 Article ID 179250.

[22] Matsushita K, Cha EK, Matsumoto K, Baba S, Chromecki TF, Fajkovic Hetal. Immunohistochemical biomarkers for bladder cancer prognosis. Int J Urol 2011; 18: 616-629.

[23] Luo Y, Zhang X, Mo M, Tan Z, Huang L, Zhou H, et al. High Ki-67 immunohistochemical reactivity correlates with poor prognosis in bladder carcinoma: A comprehensive metaanalysis with 13,053 patients involved. Medicine (Baltimore) 2016; 95: e3337.

[24] Turk NS, Aybek Z, Duzcan E. Ki-67, p53, Bcl-2 and bax expression in urothelial carcinomas of urinary bladder. Turk $\mathrm{J}$ Pathol 2010; 26: 25-30. 